Valoración económicalambiental, producción de biómasa y carbono de un bosque nativo andino, frente a plantaciones forestales eucalyptus globulus y pinus patula en la

Provincia de Loja.

Environmental economic assessment, biomass and carbon production of an andean native, forest in front of eucalyptus globulus and pinus patula forest plantations, in the province of Loja.

DOI: https://doi.org/10.29166/revfig.v1i1.1801

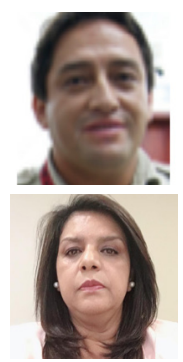

\title{
Jaramillo James
}

Funcionario Metro de Quito

james.jaramillo@metrodequito.gob.ec

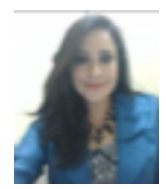

Palacios Cabrera Teresa

Docente FIGEMPA

tapalacios@uce.edu.ec

\section{Chamorro Sonia}

Docente FIGEMPA

sechamorro@uce.edu.ec

\section{RESUMEN}

En la investigación desarrollada sobre la valoración económica ambiental, producción de biomasa y carbono de un bosque nativo en la ciudad de Loja, se realizó una comparación con plantaciones forestales de Eucalyptus globulus y Pinus Patula; a través de un estudio se logró determinar que los bosques andinos nativos presentan un valor económico ambiental de 77783,4 USD/ha, el mismo que es superior al de las plantaciones forestales que tienen 46344,1 USD/ha y 45471,9 USD/ha. Por otro lado, también se encontró que la producción de biomasa es netamente superior en las plantaciones forestales que, en los bosques nativos (E. globulus 492,59 ton/ha, P. patula 333,8 ton/ha y Bosques nativos 151,91 ton/ha), con cantidades que superan más del 50\% y que, además se ve reflejado en el almacenamiento de carbono, ya que las plantaciones forestales al tener mayor producción de biomasa, también retinen mayor cantidad de carbono (E. globulus con 246,30 ton/ha, P. patula con 166,93 Bosque y andino 75,96 ton/ha).

PALABRAS CLAVE: valoración económica; biomasa; plantaciones forestales; bosques nativos; valoración ambiental.

\section{ABSTRACT}

In the research carried out on the environmental economic valuation, biomass and carbon production of a native forest in the city of Loja, a comparison was made with forest plantations of Eucalyptus globulus and Pinus Patula; Through a study it was possible to determine that native Andean forests have an economic environmental value of 77,783.4 USD / ha, which is higher than forest plantations that have 46344.1 USD / ha and 45,471.9 USD / ha. On the other hand, it was also found that biomass production is clearly higher in forest plantations than in native forests (E. globulus 492.59 tons / ha, P. patula 333.8 tons / ha and Native forests $151,91 \mathrm{t} / \mathrm{ha}$ ), with amounts that exceed more than $50 \%$ and which is also reflected in carbon storage, since forest plantations have higher biomass production also retain more carbon (E. globulus with 246, 30 ton / ha, P. patula with 166.93 Forest and Andean 75.96 tons / ha).

KEYWORDS: economic valuation; biomass; forest plantations; native forests; environmental assessment.

\section{INTRODUCCIÓN}

La biomasa es aquel componente orgánico de origen vegetal o animal, además, sirve como un parámetro para caracterizar la capacidad de los ecosistemas en acumular materia orgánica a lo largo del tiempo (Brown, 1997), está compuesta por el peso de la materia orgánica aérea y subterránea que existe en un medio forestal. Asimismo, según IPCC (2017) es la masa total de organismos vivos presentes en una área o volumen dados, se suele considerar biomasa muerta al material vegetal muerto recientemente. La biomasa es importante, pues permite cuantificar la cantidad de nutrientes en diferentes partes de las plantas y estratos de la vegetación, también facilita comparar distintos tipos de especies y vegetación, en diferentes 
sitios. Además, la cuantificación de la biomasa y el crecimiento de la vegetación en los ecosistemas son críticos para las estimaciones de fijación de carbono, un tema actualmente relevante, por sus implicaciones en el cambio climático. (Begon, Harper, Towsend, \& Riba Rovira, 1996; Brown, 1997; Dixon et al., 1994). Existen dos métodos comúnmente usados para estimar la cantidad de biomasa que son: el método directo y el indirecto. Dentro del primero está el destructivo, que consiste en cortar el árbol y determinar la biomasa pesando directamente cada componente (Klinge \& Herrera, 1983). Dentro de los indirectos se utilizan métodos de cubicación, del árbol donde se suman los volúmenes de madera, se toman muestras de ésta y se pesan en el laboratorio para calcular los factores de conversión de volumen a peso seco, es decir, la gravedad o densidad específica (M. Segura, 1997). Otra forma de estimar la biomasa es mediante ecuaciones o modelos basados en análisis de regresión, que utilizan variables colectadas en el campo, tales como: el diámetro a la altura del pecho (d), la altura comercial (hc) y total (ht), el crecimiento diamétrico, el área basal y la densidad específica de la madera; este método no es destructivo y es extrapolable a situaciones de crecimiento similares (Brown, 1997).

Los modelos de biomasa han aumentado en los últimos años y, posiblemente existen más los desarrollados para árboles individuales que para bosques. Estas ecuaciones facilitan la toma de decisiones y permiten la estimación de biomasa y carbono a gran escala (M. Segura, 1997). Dada la importancia de los bosques como reguladores del cambio climático, y la necesidad de poder estimar el carbono almacenado en los

proyectos forestales (Pérez Cordero \& Kanninen, 2003; Milena Segura \& Kanninen, 2005).

En el presente estudio, se realizó una investigación cuyo objetivo era cuantificar la producción de biomasa para bosques nativos y plantaciones forestales, así como también, realizar la valoración económica ambiental de los mismos.

Como aporte de este trabajo se evidenciará la eficiencia, desde los puntos de vista económico, social y ambiental de los bosques, frente a las plantaciones, a través de la producción de biomasa y almacenamiento de carbono, y su valoración económica ambiental.

\section{MATERIAL Y MÉTODOS.}

\section{Delimitación de la zona de estudio}

El desarrollo de este trabajo de investigación se ejecutó, desde enero a abril del 2018 en los bosques nativos y plantaciones ubicadas en la zona andina del cantón Loja, en el sector Uritusinga, Villonaco, Taquil.

Se delimitó la población, basado en un rango de altitud, el mismo que está entre 2200-2900 msnm; las plantaciones y bosques, fuera de este límite, no se consideraron para el estudio.

\section{Materiales y equipos}

Las herramientas y equipos que se utilizó para la toma de datos en el campo son: cinta diamétrica (medir diámetro), hipsómetro suunto (medir altura de árboles), podadora de aérea y de mano, segueta y balanza analógica. Además, para realizar el proceso de información y toma de datos en el laboratorio, se usó balanzas electrónicas de precisión y una estufa, la cual fue utilizada para secar las muestras.

Determinación del valor económico ambiental de los servicios y bienes del bosque nativo andino y plantaciones forestales (Pinus patula y Eucalyptus globulus).

Con la finalidad de obtener el valor económico ambiental de los tres tipos de cobertura, se trabajó con la metodología propuesta por el Ministerio del Ambiente del Ecuador, la misma que está basada en los servicios y bienes que proporcionan los ecosistemas.

\section{Servicios}

\section{- Captura de carbono}

$$
Y_{c}=\sum_{i=1}^{n} P_{c} Q_{i}^{c} N_{i}^{c}
$$

\section{Dónde}

Yc : Ingresos por la fijación del carbono (\$/año).

$P \&$ : Precio del carbono fijado (\$/ton).

$Q_{i}^{c}$ : Volumen del carbono fijado (ton/ha/año).

$\mathrm{N}$ : Número de hectáreas reconocidas para fijación de carbono.

i : Tipo de bosque considerado para el servicio de fijación de gases con efecto invernadero.

\section{Belleza escénica}

Para determinar el costo de la belleza escénica, se tomó como referencia las visitas al Parque Nacional Podocarpus en el sector Cajanuma (ubicado cerca de la zona del sector de Uritusinga), que según 
estadísticos del MAE (Ministerio del Ambiente) en el año 2017 ingresaron 9475 turistas de los cuales 7379 son nacionales y 2078 extranjeros. Además, se aplicó una encuesta a 30 turistas nacionales y extranjeros sobre su disposición a pagar, la misma que fue realizada en el puesto de control de ingreso al área.

El resultado obtenido de la encuesta, facilito la obtención de datos referentes al costo que los turistas nacionales y extranjeros estarían dispuestos a pagar, para conocer las áreas turísticas.

Para determinar el costo de la belleza escénica se utilizó la ecuación 2.

$$
Y_{b e}=P_{b e}^{E} Q_{b e}^{E}+P_{b e}^{N} Q_{b e}^{N}
$$

\section{Dónde}

$Y_{\text {be }}$ : Ingreso por belleza escénica en turismo (\$/año)

$P$ : Valor monetario pagado por turistas extranjeros

$E$
be

$\mathrm{P}_{\mathrm{be}}^{\mathrm{N}}$ : Valor monetario pagado por turistas nacionales

$E$
be persona/año)

$Q$ :Cantidad de turistas extranjeros (persona/año)

$\mathrm{Q}_{\mathrm{be}}^{\mathrm{N}}:$ :Cantidad de turistas nacionales (persona/año)

\section{Bienes}

\section{- Agua}

Para la valoración del agua, primero se determinó la oferta hídrica de cada uno de los tipos de cobertura vegetal estudiados (bosque nativo andino, plantación de pino, plantación de eucalipto). Para ello, primeramente, se utilizó los datos de la estación meteorológica la Argelia, que está ubicada cerca al proyecto a 2160 msnm, cuyo código es M033.

Luego, en base a la actividad económica más importante de la zona del proyecto que es la ganadería, se procedió a determinar el costo del agua como insumo para la producción. Para lo cual se utilizó la ecuación 3.

$$
\sum_{i=1}^{n} P_{a} Q_{i a-1}
$$

Ec.

\section{Dónde}

$Y_{a}$ : Ingreso por el aprovechamiento del agua como insumos (\$/año).

$P_{a}$ : Precio del agua como insumo de la producción
$(\$ / m 3)$.

$Q_{\text {ia-1: }}$ : Demanda de agua en el sector i(m3/año).

\section{Productos maderables}

Para este cálculo se utilizó la ecuación 4.

$$
Y_{m}=\sum_{i=1}^{n} P_{i}^{m m} Q_{i}^{m m}
$$

Ec.

\section{Dónde}

$Y_{m}$ : Ingresos por el aprovechamiento de productor maderables y no maderables (\$/año)

$P$ : Precio del bien i $(\$ / m 3)$

$\mathrm{Q}_{i}^{\mathrm{mm}}$ : Volumen del bien i (m3/año)

$i_{i}^{m m}$ Determinar el potencial de producción de biomasa y carbono en el bosque nativo andino y plantaciones forestales (Pinus patula y Eucalyptus globulus).

\section{Medición de las variables de árboles en pie y toma de muestras.}

En primer lugar, se midió el diámetro del árbol a la altura de pecho (DAP), la altura comercial $(\mathrm{Hc})$ y altura total del fuste $(\mathrm{Ht})$.

El DAP se midió con cinta diamétrica, utilizando como herramienta de precisión una barra de 1,30 $\mathrm{m}$ de largo. La altura total y comercial se midió con hipsómetro suunto.

En la figura 1, se especifican las variables usadas y que fueron medidas para el desarrollo de este trabajo investigativo.

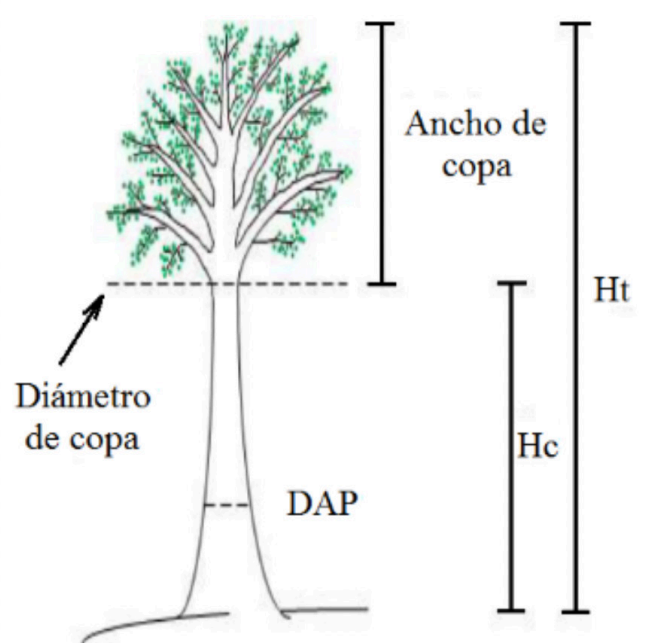

Figura 1. Variables medidas en los árboles. (Ministerio del Ambiente, 2012) 
Determinación del número de unidades de muestreo a medir por estrato

La determinación de la biomasa se la realizó en los diferentes compartimentos del bosque. Para el estrato arbóreo se midió individuos mayores a $10 \mathrm{~cm}$ de DAP, dentro de ellos, para el estrato arbustivo, se estableció dos parcelas una de $5 \times 5 \mathrm{~m}$ y otra de 2 x $2 \mathrm{~m}$ para el estrato Herbáceo y para el caso de Necromasa se estableció solo una de $2 \times 2 \mathrm{~m}$.

\section{Biomasa arbórea}

Dentro de la superficie de cada parcela se midió el DAP de 1,30 m del fuste (con lo cual se puede calcular el área basal que comprende la zona de cualquier sección transversal del fuste de un árbol, y la altura del fuste de cada árbol. Estos datos, junto con el factor de forma, permitieron calcular el volumen del fuste del árbol con la ecuación 5

\section{Dónde}

$$
V F=G^{\star} H F^{\star} F f
$$

VF: Volumen del fuste

HF: Altura del fuste

Ff: Factor de forma

G: Área Basal

El área basal se puede encontrar por medio de la ecuación 6.

\section{Dónde}

$$
\mathrm{G}=0,7854(\mathrm{DAP})^{2}
$$

Ec. (6)

\section{DAP: Diámetro a la altura de pecho de un árbol}

Biomasa de la vegetación del sotobosque (estrato arbustivo y herbáceo) y de fermentos

Cada parcela se dividió en dos zonas de $25 \mathrm{~m}^{2}$ para arbustos y de $4 \mathrm{~m}^{2}$ para las hierbas, en donde se cortó, colectó y peso toda vegetación. Además, se recogió toda la masa en el proceso de fermentación, hasta llegar al suelo mineral (necromasa). Se pesaron las dos masas y se llevaron submuestras representativas, de cada una, al laboratorio para establecer la relación peso seco sobre peso verde (Ps/Pv). El promedio de la masa seca se usó para calcular la misma en toda una hectárea, tanto de sotobosque como de fermentos.

\section{Suelos}

En dos parcelas, de cada cobertura, se hizo una calicata y se midió el primer horizonte orgánico, del cual se obtuvo una muestra mixta de $500 \mathrm{~cm}^{3}$ $\left(0,0005 \mathrm{~m}^{3}\right)$. Además, de los primeros $10 \mathrm{~cm}$ de suelo se obtuvo carbono, en base al contenido de materia orgánica, utilizando la fórmula de (Aguirre \& Aguirre, 2004), la misma que se especifica en la ecuación 7.

Dónde

$$
\mathrm{C}_{\text {suelo }}=\mathrm{Q}_{\text {MOsuelo }}{ }^{\star} 0,58
$$

$\mathrm{C}_{\text {suelo }}$ : Cantidad de carbono del suelo.

$\mathrm{Q}_{\text {Mosuelo }}$ : Cantidad de materia orgánica del suelo (en gramos o en porcentaje)

Determinar el potencial de producción de carbono del bosque andino y plantaciones forestales (Pinus patula y Eucalyptus globulus), en la zona andina del cantón Loja, provincia de Loja.

Para determinar el potencial de producción de carbono se utilizó la ecuación 8, planteada por Mireles, Carrillo y Díaz (2008). (Mireles, Carrillo, \& Diaz, 2008).

\section{Dónde}

$$
\mathrm{C}=0.5^{\star} \text { Biomasa } \quad \text { Ec. ( } 8 \text { ) }
$$

\section{C: Carbono}

\section{RESULTADOS}

Determinación del valor económico ambiental de los servicios y bienes del bosque andino y plantaciones forestales (Pinus patula y Eucalyptus globulus)

\section{Servicios \\ - Captura del carbono}

En la Tabla 1 se muestra el valor económico con el precio del mercado en la región.

Tabla 1. Valor económico por hectárea por el servicio de las coberturas bosque nativo andino, plantación de Pinus patula y de Eucalyptus globulus.

\begin{tabular}{|c|c|c|c|c|}
\hline $\begin{array}{c}\text { Tipo de } \\
\text { cobertura }\end{array}$ & $\begin{array}{c}\text { Biomasa } \\
\text { (ton/ha) }\end{array}$ & $\begin{array}{c}\text { Carbono } \\
\text { almacenado }\end{array}$ & $\begin{array}{c}\text { Precio de } \\
\text { tonelada } \\
\text { de carbono } \\
\text { mercado (USD) }\end{array}$ & $\begin{array}{c}\text { Valor } \\
\text { económico } \\
\text { por } \\
\text { hectárea }\end{array}$ \\
\hline $\begin{array}{c}\text { Bosque } \\
\text { nativo }\end{array}$ & 151,91 & 75955,00 & 81,53 & 6190,33 \\
\hline P. patula & 333,86 & 166,93 & 81,5 & 45471,9 \\
\hline E. globulus & 492,59 & 246,30 & 81,5 & 46344,1 \\
\hline
\end{tabular}

\section{Belleza escénica}

Existe una mejor disposición por parte de turistas nacionales, para pagar por el disfrute del bosque andino con un valor económico promedio de $\$ 6.06$, seguido de las plantaciones de Pinus patula (en Loja existe el Parque recreacional Pucará con esta especie) dando un valor económico promedio de $\$ 1,44$ y, por último en el menor valor la plantación de E. globulus con $\$ 0,78$. 
Los turistas extranjeros tienen mayor disponibilidad a pagar por el disfrute de los recursos escénicos con un valor económico promedio de \$10,5 seguido de las plantaciones de P. patula con 6.83 y E. globulus con $\$ 5$.

\section{Bienes}

\section{- Agua}

El volumen de oferta hídrica para bosques, plantación de P. patula y E. globulus es de 3480,36; 2610,27 y $2175,22 \mathrm{~m}^{3} / \mathrm{ha} / \mathrm{año}$, respectivamente. Esta es la cantidad de agua utilizable para las diferentes actividades que realizan las familias del área de influencia del proyecto.

Con base a los resultados mostrados, en el párrafo anterior, se determinó el valor económico de las distintas coberturas vegetales estudiadas que dieron valores de 218,1 \$/año, 163,1 \$/año y 135,9 \$año para el bosque andino, plantación de P. patula y plantación de E. globulus respectivamente.

\section{- Productos maderables}

El valor económico total por la producción de madera de los diferentes tipos de cobertura estudiados es: de la plantación de E. globulus (\$9989,5), y P. patula $(\$ 6885,6)$ y la producción del bosque andino nativo $(\$ 3639,6)$.

\section{- Productos no maderables}

En base a los inventarios en el sotobosque, se identificó y cuantificó productos forestales no maderables. En el bosque nativo se encontró especies potenciales como orquídeas Oncidium macranthum con una densidad que ocupa $1500 \mathrm{~m}^{2}$.

Se encontraron 12 tipos de orquídeas y en promedio 80 por hectárea. El Valor económico que se determinó por hectárea es de \$1200.

Determinación del potencial de producción de biomasa y carbono en el bosque andino y plantaciones forestales (Pinus patula. y Eucalyptus globulus)

\section{Determinación de biomasa}

\section{- Bosque nativo}

La producción de biomasa del bosque nativo andino es de 151.91 ton/ha.

\section{- Plantación de Pinus patula}

La producción de biomasa de la plantación de P. patula es de 333,86 Ton/ha.

\section{- Plantación de Eucayptus globulus}

La producción de biomasa de la plantación E. globulus es de 492,59 ton/ha.

\section{Determinación de carbono}

- Producción de carbono en los diferentes tipos de cobertura en estudio

Los resultados de carbono que almacena cada cobertura vegetal son: E. globulus (246,30 ton/ha), P. patula (166,93 ton/ha) y bosque 75,96 ton/ha.

Este resultado se debe a que las plantaciones presentan diámetros homogéneos, mientras que en el bosque nativo existen variadas clases diamétricas. A esto se suma que, posiblemente los bosques andino nativos del sector Uritusingan fueron sometidos a procesos de extracción selectiva de madera hace varias décadas.

\section{- Producción de carbono en el suelo en los diferentes tipos de cobertura en estudio}

Con la finalidad de homogenizar y considerando que en el campo se tomó las muestras de los primeros $10 \mathrm{~cm}$ de profundidad consideradas para el cálculo, por lo que la cantidad de suelo por hectárea es de $1000 \mathrm{~m}^{3}$. En la tabla 2 se muestran los resultados obtenidos.

Tabla 2. Producción total de carbono a en $10 \mathrm{~cm}$ suelo en bosque andino, plantación de P. patula y plantación de E. globulus.

\begin{tabular}{|c|c|c|c|c|c|}
\hline $\begin{array}{c}\text { Tipo de } \\
\text { cobertura }\end{array}$ & $\begin{array}{c}\text { Carbono } \\
(\%)\end{array}$ & $\begin{array}{c}\text { Cantidad } \\
\text { de suelo } \\
\text { orgánico } \\
\text { por } \\
\text { hectárea } \\
\left(\mathbf{m}^{\mathbf{3}}\right)\end{array}$ & $\begin{array}{c}\text { Carbono } \\
\text { total en el } \\
\text { suelo por } \\
\text { hectárea } \\
\left.\mathbf{( m}^{\mathbf{3}}\right)\end{array}$ & $\begin{array}{c}\text { Densidad } \\
\text { promedio } \\
\text { de suelos } \\
\text { ton/m }\end{array}$ & $\begin{array}{c}\mathbf{3} \\
\text { Carbono } \\
\text { total } \\
\text { ton/ha }\end{array}$ \\
\hline $\begin{array}{c}\text { Bosque } \\
\text { andino }\end{array}$ & 16,24 & 1000 & 162,4 & 2,65 & 430,36 \\
\hline P. patula & 8,41 & 1000 & 84,1 & 2,65 & 222,87 \\
\hline $\begin{array}{c}E . \\
\text { globulus }\end{array}$ & 3,19 & 1000 & 31,9 & 2,65 & 84,54 \\
\hline
\end{tabular}

\section{DISCUSIÓN}

Determinación del valor económico ambiental de los servicios y bienes del bosque andino y plantaciones forestales (Pinus patula y Eucalyptus globulus)

\section{Bienes}

Se ha logrado encontrar los valores económicos de servicios y bienes para tres coberturas vegetales y se pudo evidenciar que el valor económico ambiental del bosque nativo andino (77783,4 \$/ha) supera al de las plantaciones de Eucalyptus globulus (46344,1 $\$ /$ ha) y Pinus patula $(45471,9 \$ / \mathrm{ha})$, resultados que se dan debido a que en el bosque andino se tiene mayor índice de almacenado de carbono pero la producción de biomasa es baja con respecto a las plantaciones.

El valor económico encontrado de la belleza escénica, el mismo que fue determinado mediante una encuesta desarrollada a las distintas personas (nacionales y extranjeros) que visitan las plantaciones y bosque andino, indica que se tiene mayor apertura 
con los visitantes nacionales ya que vienen en mayor cantidad; sin embargo, los extranjeros ofrecen mejores valores de pago, es así, que el valor económico por el disfrute de los tipos de cobertura, en estudio, el que presenta mayor valor económico es el bosque andino con \$66535,74 seguido de la plantación de pino con $\$ 24818,5$ y por último la plantación de eucalipto con \$16145,62 por año.

\section{Servicios}

El volumen de oferta hídrica para bosques supera a las plantaciones. Mientras que el valor económico total por la producción de madera de los diferentes tipos de cobertura estudiado es mayor en la plantación de E. globulus $(\$ 9989,5)$, que en la plantación de P. patula $(\$ 6885,6)$ y la producción del bosque andino nativo $(\$ 3639,6)$. Estos valores corresponden al promedio de mercado que tienen los tablones y las pilas de madera, que implica los valores de procesamiento de la madera y mano de obra empleada en el aprovechamiento del bosque.

Determinación del potencial de producción de biomasa y carbono en el bosque andino y plantaciones forestales (Pinus patula. y Eucalyptus globulus)

\section{Determinación de biomasa}

La producción de biomasa es netamente mayor en las plantaciones con respecto al bosque andino, cabe recalcar, que los valores encontrados de producción de biomasa en esta investigación presentan similitudes con otros estudios y que se especifica a continuación:

- La producción de biomasa de la cobertura de bosque nativo es de 151.91 ton/ha cantidad que se asemeja mucho a los datos encontrados por Aguirre, N. (1999) en un bosque similar (148,1 ton/ha) y el por el Ministerio del Ambiente (2015), registra para ecosistemas similares 123,1 ton/ha.

- La producción de biomasa de la plantación de P. patula es de 333,86 Ton/ha; Mireles, A. et al. (2008), registro 301,9 Ton/ha, presenta valores relativamente similares, que pueden variar en función del tipo de manejo o densidad de las Plantaciones en comparación.

- La producción de biomasa de la plantación E. globulus es de 492,59 ton/ha; González et al. (2012), en plantaciones de la misma especie registraron entre 142 - 426 ton/ha, presentando valores cercanos, y la variación depende de la densidad, manejo y edad de las plantaciones, en comparación.

\section{Determinación del carbono}

Al comparar la cantidad de carbono almacenado en el bosque de Uritusinga (75,96 ton/ha), los valores son relativamente bajos, puesto que, el Ministerio del Ambiente (2015) define para los bosques siempreverde andino de Ceja Andina 105 ton/ha, y el bosque siempre verde Andino de pie de monte con 122,8 ton/ha, esta diferencia puede haberse generado por los procesos de extracción selectiva al que fueron sometidos estos bosques, hace varias décadas.

De otro lado al comparar la cantidad de carbono almacenado en la Plantación de P. patula (166,93 ton/ha) con otros bosques de $\mathrm{P}$. radiata en Chimborazo y Pichincha presentan valores de 195 ton/ ha y 284 ton/ha (Condoy \& Imaicela 2001). Se observa que la producción es relativamente parecida, sin embargo, las diferencias pueden deberse al tipo de manejo al que fueron sometidas las plantaciones.

Como se pude apreciar en la tabla 2, el suelo que almacena mayor cantidad de carbono corresponde al bosque nativo andino (430,36 ton/ha), mientras que las plantaciones de P. patula (222,87 ton/ha) y E. globulos (84,54 ton/ha) son inferiores. Cabe recalcar que la que presenta menor contenido de carbono, en el suelo, es la cobertura de eucalipto.

En estudios realizados en Costa Rica, en bosques maduros, se señala que se han registrado 411,9 toneladas por hectárea en el suelo, que es un valor relativamente similar al obtenido en los bosques en el presente estudio (430,36 ton/ha), las diferencias pueden haberse generado debido a los tipos de ecosistemas en comparación, tipo de suelo y especies que crecen en los bosques comparados. Aguilar (2011) reporta para bosques en proceso de sucesión mayores a 30 años de recuperación 109,24 y 92,29 ton/ha, siendo alto la cantidad de carbono encontrado en el suelo de la cobertura bosque andino del área de estudio.

Por otro lado, Canol y Correa (2007) en plantaciones de pino, registraron valores de carbono en el suelo de 25,95 y 43,5 ton/ha, al comparar con los resultados obtenidos en la presente investigación se evidencia que los valores registrados son altos tanto en las plantaciones de P. patula como en las de E. globulos.

\section{CONCLUSIONES}

El valor económico ambiental es mayor en el bosque nativo andino (77783,4 \$/ha) en comparación con las plantaciones de Eucalyptus globulus $(46344,1 \$ / \mathrm{ha})$ y Pinus patula (45471,9 \$/ha).

Los bienes, como madera, presentan mayor valor económico en las plantaciones que en el bosque nativo andino. Donde la cobertura de bosque nativo tiene un 
valor de \$3168, la plantación de P. Patula con \$ 6633.1 yE. globulus con $\$ 9241.7$. Estos valores corresponden a la venta de madera en tablones.

Los servicios como el disfrute de belleza escénica presentan mayor valor económico en el bosque nativo que en las plantaciones de P. patula y E. globulus. Con valores donde los turistas nacionales pagarían un promedio de $\$ 6,06$ por visitar un bosque nativo andino, seguido de las plantaciones de pino con $\$ 1,44$ y por último las plantaciones de eucalipto, donde pagarían únicamente \$0,78.

Los turistas extranjeros tienen mayor disponibilidad de pagar, por el disfrute de los recursos escénicos con valores de $\$ 10,5, \$ 6,83$ y $\$ 5$ dólares por visitar el bosque andino y las plantaciones de P. patula y E. globulus, respectivamente.

La producción de biomasa es mayor en las plantaciones de E. globulus (492,59 ton/ha), P. patula (333,8 ton/ha) en relación al bosque nativo andino (151,91 ton/ha). Los datos registrados mediante la presenteinvestigaciónfueron comparados conestudios realizados anteriormente y presentan similitud. Siendo importante indicar que las plantaciones investigadas corresponden a bosques adultos, los mismos que se han estabilizado en la generación de biomasa.

Entre los diferentes tipos de cobertura, en estudio, el compartimiento fuste es el que presentó mayor diferencia en producción de biomasa. Siendo el fuste el componente de mayor proporción en todos los estratos estudiados. El almacenamiento de carbono es mayor en las plantaciones (E. globulus con 246.3 ton/ ha, P. patula 166.93 ton/ha) que en el bosque nativo andino 75.96 ton/ha. El almacenamiento de carbono en el suelo es mayor en el bosque andino (430,36 ton/ha) que las plantaciones de P. patula (222,87 ton/ha) y E. glóbulos (84,54 ton/ha), y entre plantaciones es mayor el contenido de carbono en el suelo en la P. patula.

\section{RECOMENDACIONES}

Es importante realizar un muestreo con mayor número de parcelas para cada componente, con el fin de elevar la precisión y confiabilidad de los resultados del contenido de biomasa, de carbono que este bajo y sobre el suelo.

Es necesario realizar monitoreo de biomasa y carbono en parcelas permanentes de muestreo, para obtener datos reales del flujo de carbono en el bosque en relación con las plantaciones.

Se debería incluir dentro de las valoraciones económicas ambientales el servicio de almacenamiento de carbono que tienen los bosques, puesto que es mucho mayor que las plantaciones.

\section{REFERENCIAS}

Aguirre, N., \& Aguirre, Z. (2004). Guía para monitorear la Biomasa y dinámica de carbono en ecosistemas forestales en el Ecuador. Revista Herbario Loja No 11. Recuperado a partir de https://www.researchgate.net/publication/263684944_ Guia_para_monitorear_la_Biomasa_y_dinamica_de_ carbono_en_ecosistemas_forestales_en_el_Ecuador.

Begon, M., Harper, J. L., Towsend, C. R., \& Riba Rovira, M. (1996). Ecología: individuos, poblaciones y comunidades. Ediciones Omega. Recuperado a partir de http://www.ediciones-omega.es/ecologia/48ecologia-978-84-282-1152-9.html.

Brown, S. (1997). Estimating Biomass and Biomass Change of Tropical Forests: A Primer. Food and Agriculture Organization of the United Nations.

Dixon, R. K., Solomon, A. M., Brown, S., Houghton, R. A., Trexier, M. C., \& Wisniewski, J. (1994). Carbon Pools and Flux of Global Forest Ecosystems. Science (New York, N.Y.), 262(5134), 725-6. https://doi.org/10.1126/ science.262.5134.725.

Klinge, H., \& Herrera, R. (1983). Phytomass structure of natural plant communities on spodosols in southern Venezuela: The tall Amazon Caatinga forest. Vegetatio, 53(2), 65-84. https://doi.org/10.1007/BF00043025.

Ministerio del Ambiente. (2012). Proceso metodológico para el desarrollo de modelos alométricos para especies, Grupo de especies y estratos de bosques del Ecuador.

Mireles, M., Carrillo, F., \& Díaz, M. (2008). Determinación del carbono total en bosques mixtos de Pinus patulaSchl. et Cham. Revista Terra Latinoamericana. 27:105-114.

Pérez Cordero, L., \& Kanninen, M. (2003). Heartwood, sapwood and bark content, and wood dry density of young and mature teak (Tectona grandis) trees grown in Costa Rica. Silva Fennica, 37(1). https://doi.org/10.14214/sf.511

Segura, M. (1997). Valoración del servicio de fijación y almacenamiento de carbono en bosques privados en el área de conservación cordillera volcánica central. Universidad Nacional. Heredia (Costa Rica).

Segura, M., \& Kanninen, M. (2005). Allometric models for tree volume and total aboveground biomass in a tropical humid forest in Costa Rica. Biotropica, 37(1), 2-8. https:// doi.org/10.1111/j.1744-7429.2005.02027.x 